\title{
A Giant Pulmonary Hydatid Cyst in a 13-Year-Old Child: A Case Report
}

\author{
Nora Bigdeli ${ }^{1}$, Fatemeh Zahra Bagheri ${ }^{2}$, Fatemeh Pouladkhay ${ }^{2}$, Tayebeh Azarmehr ${ }^{3}$ and Mehdi \\ Abbasi Sahebi ${ }^{4,}{ }^{*}$ \\ ${ }^{1}$ Department of Surgery, Taleghani Children's Hospital, Golestan University of Medical Sciences, Gorgan, Iran \\ ${ }^{2}$ Department of Operating Room, Golestan University of Medical Sciences, Gorgan, Iran \\ ${ }^{3}$ Operating Room Department, School of Nursing and Midwifery, Zahedan University of Medical Science, Zahedan, Iran \\ ${ }^{4}$ Department of Surgery, Azar Hospital, Golestan University of Medical Sciences, Gorgan, Iran \\ "Corresponding author: Department of Surgery, Azar Hospital, Golestan University of Medical Sciences, Gorgan, Iran. Tel : +98-9120084942, Email: md.abbasi56@yahoo.com
}

Received 2021 September 04; Accepted 2021 September 04.

\begin{abstract}
Hydatid disease has been introduced as a crucial health issue in the Eastern Mediterranean by the World Health Organization. Surgery is the standard gold treatment for hydatid disease. Here, we reported a case of a large hydatid cyst involving the lung in a 13-year-old girl complaining of shortness of breath and cough for 8 months. Early symptoms include fever, cough, and shortness of breath. After admission, computed tomography verified giant lung hydatid cysts. Complete collapse of the right lung and progressive respiratory distress of the patient were treated with thoracotomy considering the size of the hydatid cyst in the lung. It should be noted that in such patients, the preservation of lung tissue function is very significant, and also, the less part of the lung is removed after surgery, the fewer complications the patient will suffer.
\end{abstract}

Keywords: Children, Gland, Hydatid Cyst, Lung, Segmentectomy

\section{Introduction}

Hydatid cyst disease has been introduced as a crucial health issue in the Eastern Mediterranean by the World Health Organization (WHO) (1). It is caused by an infection with a hydatid worm Echinococcus granulosus. Human infection mainly occurs due to contact with infected dogs or eating foods contaminated with the parasite's eggs (24). The cyst can affect all organs in the body; however, the most common sites for its spread are the liver (65\% to $70 \%$ ), lungs (25\%), brain (less than $5 \%$ of cases), and bone (less than $2 \%$ of cases). Other organs are rarely affected (5). Symptoms and complications are related to the location and size of the hydatid cyst. The most common symptoms of pulmonary hydatid cyst (PHC) are cough, chest pain, shortness of breath, and hemoptysis (2-4). The spread of giant hydatid cysts with extensive pleural effusion depends on the geographical area of the habitat. Moreover, due to the risk of allergic reactions, anaphylactic shock, and its spread, aspiration should be avoided (6). There are different methods for treating hydatid cysts, such as open surgery and complete removal of the cyst, laparoscopic surgery, removal of the contents of the cyst with ultrasound interventional treatment guided by ultrasound, and filling the cavity using sclerosis drugs, and reaspiration of this drug. Albendazole is also currently used as oral treatment, which shrinks the cyst, and then, treatment with other methods continues (7). Surgery is the gold standard procedure for PHC. Large cysts that are superficial and vulnerable to rupture, infected cysts, cysts located near vital anatomical structures, and cysts that are large and significant in size are treated with surgery $(8,9)$. Because a ruptured cystic echinococcus causes severe symptoms and death, it should be considered more carefully before rupture and dangerous symptoms for the patient. In addition, the necessary measures should be taken to treat and remove it as soon as possible. Here, we reported a 13year-old girl with a giant PHC.

\section{Case Presentation}

The patient was a 13-year-old girl living in one of the villages of Kalaleh City in Golestan Province. She was referred to the emergency room of Taleghani Children Educational and Medical Center in Gorgan and had complaints 
of cough and shortness of breath, which had been progressive for eight months and had not improved despite visiting physicians and the use of multiple medications. On admission, her vital signs were as follows: Spo2: 98\%, blood pressure: $100 / 80 \mathrm{mmHg}$, pulse rate (PR): 96 , respiratory rate (RR): $24 \mathrm{bpm}$, and temperature: $37.3^{\circ} \mathrm{C}$. Decreased appetite and weight loss were also observed in her history. In the initial examination of the patient, symptoms, such as fever, cough, and shortness of breath, and in the physical examination, decreased respiratory sounds in the right lung and pulmonary voice violencewere observed. According to the patient's condition, initial tests were performed (Table 1).

\begin{tabular}{lc}
\hline Table 1. Laboratory Data of the Patient on Admission & Result \\
\hline Parameters & 14900 \\
\hline WBC, mm & 6.23 \\
\hline RBC, mil/Cumm & 11.5 \\
\hline HB, g/dL & 67.6 \\
\hline MCV, fL & 20.7 \\
\hline MCH, pg & 30.6 \\
\hline MCHC, g/dL & 0.5 \\
\hline Creatinine, mg/dL & 45 \\
\hline AST, U/L & 15 \\
\hline ALT, U/L & 30 \\
\hline ESR, mm/h & 14.4 \\
\hline PT, $\mathbf{S}$ & $1+$ \\
\hline CRP, $\mathbf{~ m g / L ~}$ & \\
\hline
\end{tabular}

In the patient's chest X-ray, severe involvement of the right lung was observed, and the presence of a mass in the patient's lung was suggested (Figure 1).

Ultrasound indicated a very large cystic mass (approximately $182 \times 142 \mathrm{~mm}$ ) on the right side of the thoracic abdominis. Based on these results, the patient was initially diagnosed with hemitorax mass. The spiral computerized tomography (CT) scan of lung mediastinum (injection) was performed, which showed an image of a unilocular cystic structure approximately $15 \times 20 \mathrm{~cm}$ in size, including the complete right hemithorax with a complete collapse of the right lung and rib media mediastinum shift, primarily suggesting an intact hydatid cyst (Figure 2). Finally, the patient was prepared for the surgery with a diagnosis of hydatid cyst. Following diagnosis, albendazole was started at a dose of $15 \mathrm{mg} / \mathrm{kg}$ body weight daily.

After general anesthesia, the patient was placed in the lateral position of the right decubitus so that the right hemithorax was raised for better vision of the surgeon. The thorax was opened from the intercostal space 6 , and a large hydatid cyst was observed. After suctioning the fluid inside the cyst, several bronchi that had opened into the cyst space were ligated. In addition, the cyst then became capitonnage. Surgical gas containing 20\% hypertonic serum was present at the site and around the cyst and the wall of the incision. Furthermore, the lower segment of the upper lobe and the upper segment of the lower lobe as part of the cyst were removed by segmentectomy. In the end, two chest tubes (22 Fr) were placed for the patient, and after making sure that the ducts were closed and there was no air leakage, the chest was closed (Figure 3).

During the operation, one unit of pack cell was injected for the patient. The pathology report completely confirmed the hydatid cyst. 7 days after the operation, the chest tubes were removed, and in the postoperative followup, the patient's general condition was completely normal; moreover, the previous restrictions were removed.

\section{Discussion}

The clinical presentation of a hydatid cyst depends on the location and size of the cyst. Small and peripheral cysts are usually asymptomatic, while large cysts may present with signs of compression of nearby organs. Most pulmonary hydatid cysts may remain asymptomatic for a long time until they are large enough to cause symptoms, such as unproductive cough, chest pain, and shortness of breath. Some complications, such as rupture, secondary infection, pneumothorax, and fatigue may precede diagnosis (10). The results of a study conducted on children with a hydatid cyst in Turkey indicated that surgical treatment is the method of choice for the treatment of hydatid cyst in children (11). During hydatid cyst resection surgery, the damaged parenchyma should be completely removed, but if the lung involvement is more than 60\%, lobectomy or segmentectomy is done $(6,12)$. In the study by Karimi et al. (13), after posterior thoracotomy, the hydatid cyst was drained (cystectomy), and then, the patient's symptoms were controlled with albendazole. Sokouti et al. (6) reported a 9-year-old child diagnosed with a pulmonary hydatid cyst was operated. Due to the involvement of a large part of the lung, a lower lobectomy of the lower right lung was performed for the patient. Furthermore, Engstrom et al. (14) used left upper lung lobectomy to remove hydatid cysts. However, in the present study, due to the involvement of the lower segment of the upper lobe and the upper 


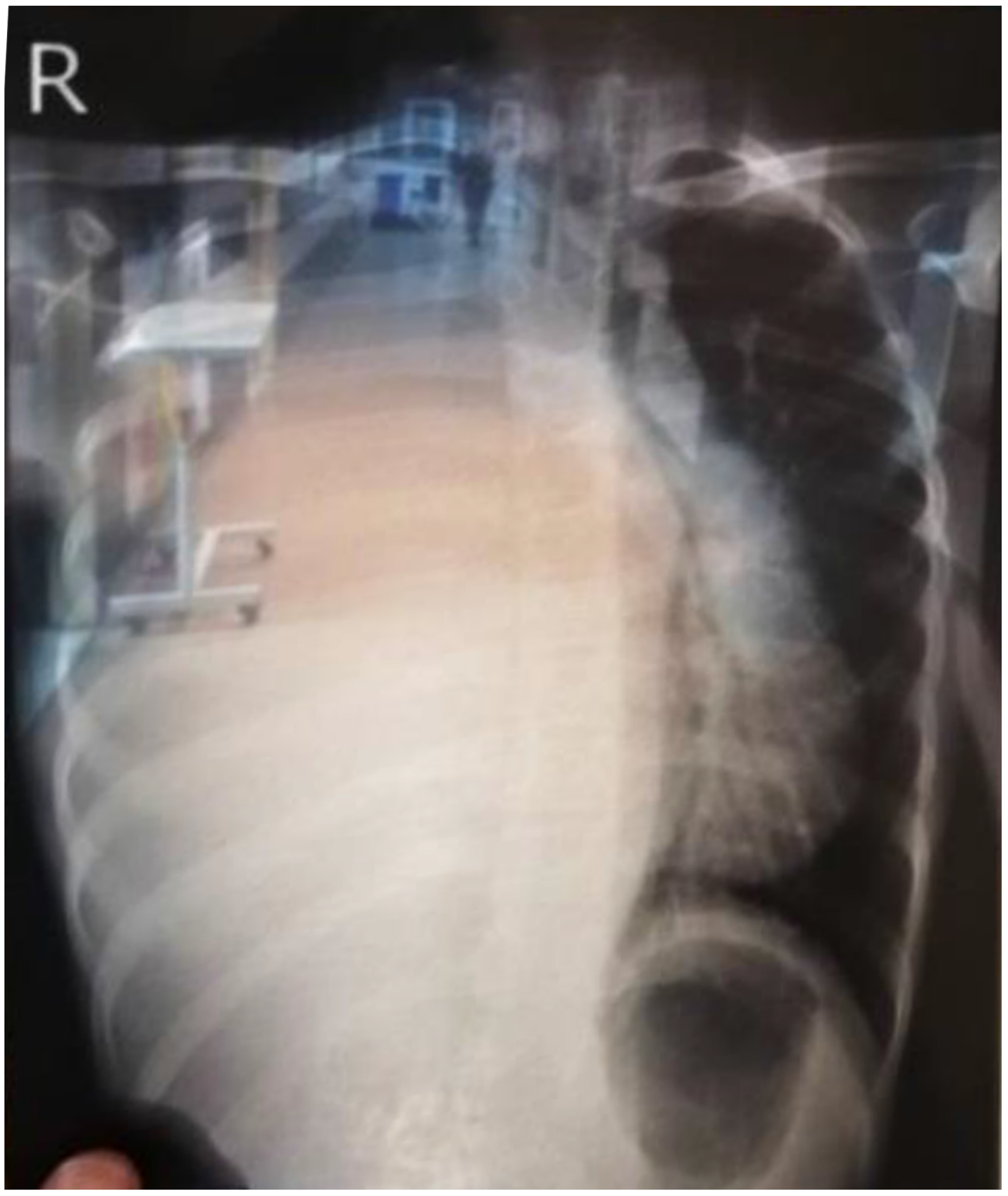

Figure 1. CXR image of the patient 




Figure 2. The patient's CT scan

segment of the lower lobe of the right lung, despite the size of the cyst and the difficulty of segmentectomy compared to other methods, segmentectomy was performed and also part of both upper and lower lobe was preserved. Segmentectomy has not been used in the previous studies, which is the difference between our study and other studies.

Finally, it should be noted that in such patients, the preservation of the lung tissue function is very important. In addition, the less part of the lung is removed after surgery, the fewer complications the patient will suffer (15). Accordingly, in dealing with similar patients, segmentation is a priority over lobectomy; however, it requires more time and precision. A segmentectomy was performed in our case to preserve the lung tissue of the patient. The limitation of this case report was that, unfortunately, due to the urgency of the operation, no photograph was taken from the patient during the operation.

\section{Acknowledgments}

The authors express their gratitude to the aforementioned patient for authorizing the publication of this report.

\section{Footnotes}

Authors' Contribution: All authors discussed the results and contributed to the preparation of the final manuscript.

Conflict of Interests: The authors declared any conflicts of interest.

Funding/Support: No financial support was received for this research.

Informed Consent: A written informed consent was obtained from the patient. 


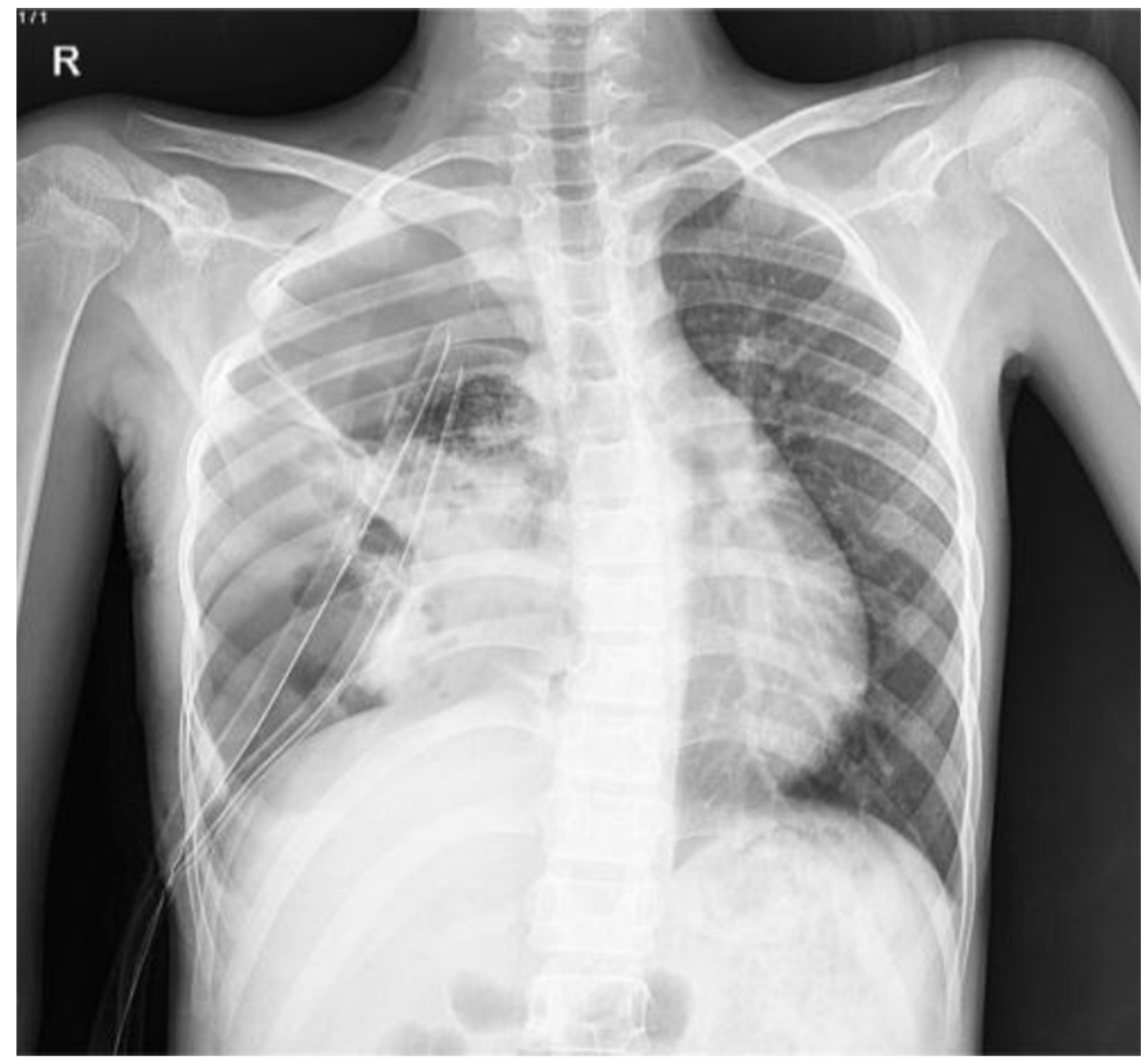

Figure 3. Photograph of the patient's chest after surgery

\section{References}

1. Mahmodlou R, Sepehrvand N, Nasiri M. Saucerization: a modified uncapitonnage method of surgery for pulmonary hydatidosis. World J Surg. 2013;37(9):2129-33. doi: 10.1007/s00268-013-2093-7. [PubMed: 23657752].

2. Arinc S, Kosif A, Ertugrul M, Arpag H, Alpay L, Unal O, et al. Evaluation of pulmonary hydatid cyst cases. Int J Surg. 2009;7(3):192-5. doi: 10.1016/j.ijsu.2008.11.003. [PubMed: 19369124].

3. Azulay AA, Refaely Y, Ruderman L, Nesher L, Semionov M. A Huge Hydatid Pulmonary Cyst. Int Med Case Rep J. 2020;13:61-4. doi: 10.2147/IMCRJ.S228657. [PubMed: 32158277]. [PubMed Central: PMC7047981].

4. Santivanez S, Garcia HH. Pulmonary cystic echinococcosis. Curr Opin Pulm Med.2010;16(3):257-61. doi: 10.1097/MCP.0b013e3283386282.
[PubMed: 20216420]. [PubMed Central: PMC3362862].

5. Székely R, Rojo M, Ramirez R. The location of hydatid cysts in livestock. Bol Chil Parasitol. 1973.

6. Sokouti M, Shokouhi B, Sokouti M, Sokouti B. Giant Pulmonary Hydatid Cyst and Trauma in a 9 Year-Old Child: A Case Report. Open Respir Med J. 2015;9:67-9. doi: 10.2174/1874306401509010067. [PubMed: 26089993]. [PubMed Central: PMC4468588].

7. Golfam F, Golfam P, Khalaj A, Sayed Mortaz S, Taheri H, Amini M. Evolution in treatment methods of hepatic hydatid cyst.J Hakim Res. 2008.

8. Rawat S, Kumar R, Raja J, Singh RS, Thingnam SKS. Pulmonary hydatid cyst: Review of literature. J Family Med Prim Care. 2019;8(9):2774-8. doi: 10.4103/jfmpc.jfmpc_624_19. [PubMed: 31681642]. [PubMed Central: PMC6820383]. 
9. McManus DP, Zhang W, Li J, Bartley PB. Echinococcosis. The Lancet. 2003;362(9392):1295-304. doi:10.1016/s0140-6736(03)14573-4.

10. Sinmaz E, Celiksoz A. A Giant pulmonary hydatid cyst treated without lobectomy. Yonsei Med J. 2009;50(6):856-8. doi: 10.3349/ymj.2009.50.6.856. [PubMed: 20046431]. [PubMed Central: PMC2796417].

11. Bakal U, Simsek S, Kazez A. Surgical and Molecular Evaluation of Pediatric Hydatid Cyst Cases in Eastern Turkey. Korean J Parasitol. 2015;53(6):785-8. doi: 10.3347/kjp.2015.53.6.785. [PubMed: 26797450]. [PubMed Central: PMC4725236].

12. Usluer O, Ceylan KC, Kaya S, Sevinc S, Gursoy S. Surgical management of pulmonary hydatid cysts: is size an important prognostic indicator? Tex Heart Inst J. 2010;37(4):429-34. [PubMed: 20844615]. [PubMed Central: PMC2929855].
13. Karimi M, Rostami A, Spotin A, Rouhani S. Ruptured pulmonary hydatid cyst: a case report. J Parasit Dis. 2017;41(3):899-902. doi: 10.1007/s12639-017-0880-z. [PubMed: 28848299]. [PubMed Central: PMC5555925].

14. Engstrom ELS, Salih GN, Wiese L. Seronegative, complicated hydatid cyst of the lung: A case report. Respir Med Case Rep. 2017;21:96-8. doi: 10.1016/j.rmcr.2017.04.005. [PubMed: 28443235]. [PubMed Central: PMC5392770].

15. Kabiri el H, Traibi A, El Hammoumi M, El Oueriachi F, Arsalane A. Parenchyma sparing procedures is possible for most pulmonary hydatid disease without recurrence and low complications. Med Arch. 2012;66(5):332-5. doi:10.5455/medarh.2012.66.332-335. [PubMed: 23097973]. 